\title{
Generalized Lacunary Statistical Difference Sequence Spaces of Fractional Order
}

\author{
Ugur Kadak \\ Department of Mathematics, Bozok University, Yozgat, Turkey \\ Correspondence should be addressed to Ugur Kadak; ugurkadak@gmail.com
}

Received 28 April 2015; Accepted 16 June 2015

Academic Editor: Binod C. Tripathy

Copyright (C) 2015 Ugur Kadak. This is an open access article distributed under the Creative Commons Attribution License, which permits unrestricted use, distribution, and reproduction in any medium, provided the original work is properly cited.

\begin{abstract}
We generalize the lacunary statistical convergence by introducing the generalized difference operator $\Delta_{\nu}^{\alpha}$ of fractional order, where $\alpha$ is a proper fraction and $\nu=\left(\nu_{k}\right)$ is any fixed sequence of nonzero real or complex numbers. We study some properties of this operator and investigate the topological structures of related sequence spaces. Furthermore, we introduce some properties of the strongly Cesaro difference sequence spaces of fractional order involving lacunary sequences and examine various inclusion relations of these spaces. We also determine the relationship between lacunary statistical and strong Cesaro difference sequence spaces of fractional order.
\end{abstract}

\section{Introduction}

By $\omega$, we denote the space of all real valued sequences and any subspace of $w$ is called a sequence space. Let $\ell_{\infty}, c$, and $c_{0}$ be the linear spaces of bounded, convergent, and null sequences $x=\left(x_{k}\right)$ with real or complex terms, respectively, normed by $\|x\|_{\infty}=\sup _{k}\left|x_{k}\right|$, where $k \in \mathbb{N}$, the set of positive integers. With this norm, it is proved that these are all Banach spaces. Also by bs, cs, $\ell_{1}$, and $\ell_{p}$, we denote the spaces of all bounded, convergent, absolutely summable, and $p$-absolutely summable series, respectively.

The concept of difference sequence space was determined by Kızmaz [1]. Et and Colak generalized difference sequence spaces [2]. Later on Et and Esi [3] generalized these sequence spaces to the following sequence spaces. Let $v=\left(v_{k}\right)$ be any fixed sequence of nonzero complex numbers and let $m$ be a nonnegative integer. Then, $\Delta_{\nu}^{m}(X)=\left\{x=\left(x_{k}\right):\left(\Delta_{\nu}^{m} x\right) \in X\right\}$ for $X=\ell_{\infty}, c$ or $c_{0}$, where $m \in \mathbb{N}, \Delta_{\gamma}^{0} x=\left(v_{k} x_{k}\right), \Delta_{\gamma}^{m} x=$ $\left(\Delta_{v}^{m-1} x_{k}-\Delta_{v}^{m-1} x_{k+1}\right)$, and so $\Delta_{v}^{m} x_{k}=\sum_{i=0}^{m}(-1)^{i}\left(\begin{array}{c}m \\ i\end{array}\right) v_{k+i} x_{k+i}$. These are Banach spaces with the norm defined by $\|x\|_{\Delta}=$ $\sum_{i=1}^{m}\left|v_{i} x_{i}\right|+\sup _{k}\left|\Delta_{\gamma}^{m} x_{k}\right|$. Furthermore Et and Basarir [4] have generalized difference sequence spaces. Aydın and Başar [5] have introduced some new difference sequence spaces. Also the notion of difference sequence has been extended by Mursaleen [6], Mursaleen and Noman [7], Malkowsky et al. [8], and Bektaş et al. [9]. Also Tripathy et al. [10, 11] have generalized difference sequences by Orlicz functions.

Let $\theta=\left(k_{r}\right)$ be the sequence of positive integers such that $k_{0}=0,0<k_{r}<k_{r+1}$, and $h_{r}=\left(k_{r}-k_{r-1}\right) \rightarrow \infty$ as $r \rightarrow \infty$. Then $\theta$ is called a lacunary sequence. The intervals determined by $\theta$ will be denoted by $I_{r}=\left(k_{r-1}, k_{r}\right]$. Das and Mishra [12] introduced lacunary strong almost convergence. Colak et al. [13] have studied lacunary strongly summable sequences. Also some geometric properties of sequence spaces involving lacunary sequence have been examined by Karakaya [14]. Et has generalized Cesaro difference sequence spaces involving lacunary sequences [15].

Besides the Cesaro sequence spaces $\mathrm{Ces}_{p}$ and $\mathrm{Ces}_{\infty}$ have been introduced by Shiue [16]. Jagers [17] has determined the Köthe duals of the sequence space $\operatorname{Ces}_{p}(1<p<\infty)$. Later on the Cesaro sequence spaces $X_{p}$ and $X_{\infty}$ of nonabsolute type are defined by $\mathrm{Ng}$ and Lee $[18,19]$.

The main focus of the present paper is to generalize strong Cesaro and lacunary statistical difference sequence spaces and investigate their topological structures as well as some interesting results concerning the operator $\Delta_{\gamma}^{\alpha}$.

The rest of this paper is organized, as follows: in Section 2, some required definitions and consequences related to the difference operator $\Delta^{\alpha}$ are given. Also some new classes of difference sequences of fractional order involving lacunary 
sequences are determined and some topological properties are investigated. Section 3 is devoted to the strong Cesaro difference sequence spaces of fractional order. Prior to stating and proving the main results concerning these spaces, we give some theorems about the notion of linearity and $B K$ space. In final section, we present some theorems related to the lacunary statistical convergence of difference sequences of fractional order and examine some inclusion relations of these spaces.

\section{Some New Difference Sequence Spaces with Fractional Order}

By $\Gamma(\alpha)$, we denote the Euler gamma function of a real number $\alpha$. Using the definition, $\Gamma(\alpha)$ with $\alpha \notin\{0,-1,-2,-3, \ldots\}$ can be expressed as an improper integral as follows:

$$
\Gamma(\alpha)=\int_{0}^{\infty} e^{-t} t^{\alpha-1} d t
$$

For a positive proper fraction $\alpha$, Baliarsingh and Dutta [20, 21] (also see [22]) have defined the generalized fractional difference operator $\Delta^{\alpha}$ as

$$
\Delta^{\alpha}\left(x_{k}\right)=\sum_{i=0}^{\infty}(-1)^{i} \frac{\Gamma(\alpha+1)}{i ! \Gamma(\alpha-i+1)} x_{k+i} .
$$

In particular, we have

(i) $\Delta^{1 / 2}\left(x_{k}\right)=x_{k}-(1 / 2) x_{k+1}-(1 / 8) x_{k+2}-(1 / 16) x_{k+3}-$ $(5 / 128) x_{k+4}-(7 / 256) x_{k+5}-(21 / 1024) x_{k+6}-\cdots$

(ii) $\Delta^{-1 / 2}\left(x_{k}\right)=x_{k}+(1 / 2) x_{k+1}+(3 / 8) x_{k+2}+(5 / 16) x_{k+3}+$ $(35 / 128) x_{k+4}+(63 / 256) x_{k+5}+(231 / 1024) x_{k+6}+\cdots$,

(iii) $\Delta^{2 / 3}\left(x_{k}\right)=x_{k}-(2 / 3) x_{k+1}-(1 / 9) x_{k+2}-(4 / 81) x_{k+3}-$ $(7 / 243) x_{k+4}-(14 / 729) x_{k+5}-(91 / 6561) x_{k+6}-\cdots$.

Theorem 1 (see [20]). (a) For proper fraction $\alpha, \Delta^{\alpha}: \omega \rightarrow \omega$ defined by (2) is a linear operator.

(b) For $\alpha, \beta>0, \Delta^{\alpha}\left(\Delta^{\beta}\left(x_{k}\right)\right)=\Delta^{\alpha+\beta}\left(x_{k}\right)$ and $\Delta^{\alpha}\left(\Delta^{-\alpha}\left(x_{k}\right)\right)=x_{k}$.

Now, we determine the new classes of difference sequence spaces $\Delta_{\nu}^{\alpha}(X)$ as follows:

$$
\Delta_{\nu}^{\alpha}(X):=\left\{x=\left(x_{k}\right) \in \omega:\left(\Delta_{v}^{\alpha} x\right) \in X\right\},
$$

where $\Delta_{\nu}^{\alpha}\left(x_{k}\right)=\sum_{i=0}^{\infty}(-1)^{i}(\Gamma(\alpha+1) / i ! \Gamma(\alpha-i+1)) v_{k+i} x_{k+i}$ and $X$ is any sequence spaces.

Theorem 2. For a proper fraction $\alpha$, if $X$ is a linear space, then $\Delta_{v}^{\alpha}(X)$ is also a linear space.

Proof. The proof is straightforward (see [20]).

Theorem 3. If $X$ is a Banach space with the norm $\|\cdot\|_{\infty}$, then $\Delta_{v}^{\alpha}(X)$ is also a Banach space with the norm $\|\cdot\|_{\Delta_{\nu}^{\alpha}}$ defined by

$$
\|x\|_{\Delta_{\nu}^{\alpha}(X)}=\sum_{i=0}^{\infty}\left|v_{i} x_{i}\right|+\left\|\Delta_{\nu}^{\alpha} x\right\|_{\infty},
$$

where $\left\|\Delta_{v}^{\alpha} x\right\|_{\infty}=\sup _{k}\left|\Delta_{\nu}^{\alpha}\left(x_{k}\right)\right|$.
Proof. Proof of this theorem is a routine verification, hence omitted.

Remark 4. Without loss of generality, we assume throughout that each series given in (4) is convergent. Furthermore, if $\alpha$ is a positive integer, then these infinite sums in (4) reduce to finite sums; that is, $\sum_{i=0}^{\alpha}(-1)^{i}(\Gamma(\alpha+1) / i ! \Gamma(\alpha-i+1)) v_{k+i} x_{k+i}$ and $\sum_{i=0}^{\alpha}\left|v_{i} x_{i}\right|$.

Lemma 5. Let $\alpha$ be a proper fraction. If $X \subset Y$, then $\Delta_{v}^{\alpha}(X) \subset$ $\Delta_{v}^{\alpha}(Y)$.

Proof. Let $X \subset Y$ and $x=\left(x_{k}\right) \in \Delta_{v}^{\alpha}(X)$. It is trivial that $\left(\Delta_{v}^{\alpha} x\right) \in X$ implies $\left(\Delta_{v}^{\alpha} x\right) \in Y$. Hence $x \in \Delta_{v}^{\alpha}(Y)$ and $\Delta_{v}^{\alpha}(X) \subset$ $\Delta_{v}^{\alpha}(Y)$.

Theorem 6. Let $X$ be a Banach space and $K$ a closed subset of $X$. Then $\Delta_{v}^{\alpha}(K)$ is also closed subset of $\Delta_{v}^{\alpha}(X)$.

Proof. By using Lemma 5, it is trivial that $\Delta_{v}^{\alpha}(K) \subset \Delta_{v}^{\alpha}(X)$. Now we prove that $\overline{\Delta_{\nu}^{\alpha}(K)}=\Delta_{\nu}^{\alpha}(\bar{K})$. Let $x \in \overline{\Delta_{\nu}^{\alpha}(K)}$; then there exists a sequence $\left(x^{n}\right) \in \Delta_{\gamma}^{\alpha}(K)$ such that

$$
\begin{aligned}
& \left\|x^{n}-x\right\|_{\Delta_{\nu}^{\alpha}(K)} \longrightarrow 0 \quad \text { as } n \longrightarrow \infty, \\
& \text { i.e. } \sum_{i=0}^{\infty}\left|v_{i}\left(x_{i}^{n}-x_{i}\right)\right| \\
& \quad+\sup _{k}\left|\sum_{i=0}^{\infty}(-1)^{i} \frac{\Gamma(\alpha+1)}{i ! \Gamma(\alpha-i+1)} v_{k+i}\left(x_{k+i}^{n}-x_{k+i}\right)\right| \\
& \quad \longrightarrow 0
\end{aligned}
$$

$$
\begin{aligned}
& \text { i.e. } \lim _{n} \sup _{k} \mid v_{k}\left(x_{k}^{n}-x_{k}\right)-\alpha v_{k+1}\left(x_{k+1}^{n}-x_{k+1}\right) \\
& +\frac{\alpha(\alpha-1)}{2} v_{k+2}\left(x_{k+2}^{n}-x_{k+2}\right)+\cdots \mid=0 .
\end{aligned}
$$

Thus, we observe that

$$
\begin{aligned}
\left\|x_{j}^{n}-x_{j}\right\|_{\Delta_{\nu}^{\alpha}(K)}= & \sum_{i=0}^{\infty}\left|v_{i}\left(x_{i}^{n}-x_{i}\right)\right| \\
& +\left\|\Delta_{v}^{\alpha}\left(x_{j}^{n}\right)-\Delta_{\nu}^{\alpha}\left(x_{j}\right)\right\|_{\infty} \longrightarrow 0,
\end{aligned}
$$

as $n \rightarrow \infty$ in $K$. This implies that $x \in \Delta_{\nu}^{\alpha}(\bar{K})$.

Conversely, let $x \in \Delta_{\gamma}^{\alpha}(\bar{K})$; then $x \in \Delta_{\gamma}^{\alpha}(K)$. Since $K$ is closed $\overline{\Delta_{\nu}^{\alpha}(K)}=\Delta_{\nu}^{\alpha}(K)$, hence $\Delta_{\nu}^{\alpha}(K)$ is a closed subset of $\Delta_{\gamma}^{\alpha}(X)$.

Theorem 7. If $X$ is a BK-space with the norm $\|\cdot\|_{\infty}$, then $\Delta_{v}^{\alpha}(X)$ is also a BK-space with the norm given in (4). 
Proof. It is clear that $\Delta_{\nu}^{\alpha}(X)$ is a Banach space (see Theorem 3). Suppose that $\left\|x_{j}^{n}-x_{j}\right\|_{\Delta_{\nu}^{\alpha}(X)} \rightarrow 0$ for each $j \in \mathbb{N}$. One can conclude that $\left|v_{j}\left(x_{j}^{n}-x_{j}\right)\right| \rightarrow 0$ as $n \rightarrow \infty$ for each $j \in \mathbb{N}$ and implies $\left\|\Delta_{\nu}^{\alpha}\left(x_{j}^{n}\right)-\Delta_{v}^{\alpha}\left(x_{j}\right)\right\|_{\infty} \rightarrow 0$ as $n \rightarrow \infty$. This follows from the fact that

$$
\begin{aligned}
\sup _{k}\left|\sum_{i=0}^{\infty}(-1)^{i} \frac{\Gamma(\alpha+1)}{i ! \Gamma(\alpha-i+1)} v_{k+i}\left(x_{k+i}^{n}-x_{k+i}\right)\right| & \longrightarrow 0, \\
& \left(v_{k+i} \neq 0\right),
\end{aligned}
$$

where $P=\sum_{i=0}^{\infty}(-1)^{i}(\Gamma(\alpha+1) / i ! \Gamma(\alpha-i+1))$; since $\alpha>0$ the series represented by $P$ is finite. Hence $\left|x_{j}^{n}-x_{j}\right| \rightarrow 0$ as $n \rightarrow$ $\infty$ for each $j \in \mathbb{N}$. Therefore $\Delta_{\gamma}^{\alpha}(X)$ is a Banach space with the continuous coordinates. This completes the proof.

Definition 8. A sequence $x=\left(x_{k}\right)$ is said to be $\Delta_{v}^{\alpha}$-strongly Cesaro convergent if there is a real or complex number $L$ such that

$$
\lim _{n \rightarrow \infty} \frac{1}{n} \sum_{k=0}^{n}\left|\sum_{i=0}^{\infty}(-1)^{i} \frac{\Gamma(\alpha+1)}{i ! \Gamma(\alpha-i+1)} v_{k+i} x_{k+i}-L\right|^{p}=0
$$

for some $L$,

where $p$ is a fixed positive number and $\alpha$ is a proper fraction. The number $L$ is unique when it exists. By $\Delta_{v}^{\alpha}\left(\omega_{p}\right)$, one denotes the set of all strongly $\Delta_{v}^{\alpha}$-Cesaro convergent sequences. In this case, one writes $x_{k} \rightarrow L\left(\Delta_{\nu}^{\alpha}\left(\omega_{p}\right)\right)$.

Theorem 9. The sequence space $\Delta_{\nu}^{\alpha}\left(\omega_{p}\right)$ is a Banach space for $1 \leq p<\infty$ normed by

$$
\begin{aligned}
\|x\|_{\Delta_{\nu_{1}}} & =\sum_{i=0}^{\infty}\left|v_{i} x_{i}\right| \\
+ & \sup _{n \in \mathbb{N}_{1}}\left(\frac{1}{n} \sum_{k=0}^{n}\left|\sum_{i=0}^{\infty}(-1)^{i} \frac{\Gamma(\alpha+1)}{i ! \Gamma(\alpha-i+1)} v_{k+i} x_{k+i}\right|^{p}\right)^{1 / p}
\end{aligned}
$$

and a complete p-normed space with the p-norm

$$
\begin{aligned}
& \|x\|_{\Delta_{v_{2}}} \\
& =\sum_{i=0}^{\infty}\left|v_{i} x_{i}\right|^{p} \\
& \quad+\sup _{n \in \mathbb{N}_{1}} \frac{1}{n} \sum_{k=0}^{n}\left|\sum_{i=0}^{\infty}(-1)^{i} \frac{\Gamma(\alpha+1)}{i ! \Gamma(\alpha-i+1)} v_{k+i} x_{k+i}\right|^{p}
\end{aligned}
$$

for $0<p<1$.

Proof. Proof follows by using Theorem 3.

\section{Cesaro Difference Sequence Spaces of Fractional Order}

In this section by using the operator $\Delta_{\gamma}^{\alpha}$, we introduce some new sequence spaces $C\left(\Delta_{v}^{\alpha}, p\right)_{\theta}, C\left[\Delta_{v}^{\alpha}, p\right]_{\theta}, C_{\infty}\left(\Delta_{v}^{\alpha}, p\right)_{\theta}$,
$C_{\infty}\left[\Delta_{\nu}^{\alpha}, p\right]_{\theta}$, and $N\left(\Delta_{\nu}^{\alpha}, p\right)_{\theta}$ involving lacunary sequences $\theta$ and arbitrary sequence $p=\left(p_{r}\right)$ of strictly positive real numbers.

We define the sequence spaces as follows:

$$
\begin{aligned}
& C\left(\Delta_{\nu}^{\alpha}, p\right)_{\theta}:=\left\{x=\left(x_{k}\right) \in \omega:\right. \\
& \left.\sum_{r=1}^{\infty}\left|\frac{1}{h_{r}} \sum_{k \in I_{r}} \sum_{i=0}^{\infty}(-1)^{i} \frac{\Gamma(\alpha+1)}{i ! \Gamma(\alpha-i+1)} v_{k+i} x_{k+i}\right|^{p_{r}}<\infty\right\}, \\
& C\left[\Delta_{\nu}^{\alpha}, p\right]_{\theta}:=\left\{x=\left(x_{k}\right) \in \omega:\right. \\
& \sum_{r=1}^{\infty}\left(\frac{1}{h_{r}} \sum_{k \in I_{r}}\left|\sum_{i=0}^{\infty}(-1)^{i} \frac{\Gamma(\alpha+1)}{i ! \Gamma(\alpha-i+1)} v_{k+i} x_{k+i}\right|\right)^{p_{r}} \\
& <\infty\},
\end{aligned}
$$

$$
\begin{aligned}
& C_{\infty}\left(\Delta_{\nu}^{\alpha}, p\right)_{\theta}:=\left\{x=\left(x_{k}\right) \in \omega:\right. \\
& \left.\sup _{r}\left|\frac{1}{h_{r}} \sum_{k \in I_{r}} \sum_{i=0}^{\infty}(-1)^{i} \frac{\Gamma(\alpha+1)}{i ! \Gamma(\alpha-i+1)} v_{k+i} x_{k+i}\right|^{p_{r}}<\infty\right\},
\end{aligned}
$$

$$
\begin{aligned}
C_{\infty}\left[\Delta_{v}^{\alpha}, p\right]_{\theta}:=\left\{x=\left(x_{k}\right) \in \omega: \sup _{r} \frac{1}{h_{r}}\right. \\
\left.\cdot \sum_{k \in I_{r}}\left|\sum_{i=0}^{\infty}(-1)^{i} \frac{\Gamma(\alpha+1)}{i ! \Gamma(\alpha-i+1)} v_{k+i} x_{k+i}\right|^{p_{r}}<\infty\right\},
\end{aligned}
$$

$$
\begin{aligned}
& N\left(\Delta_{\nu}^{\alpha}, p\right)_{\theta}:=\left\{x=\left(x_{k}\right) \in \omega: \lim _{r} \frac{1}{h_{r}}\right. \\
& \left.\cdot \sum_{k \in I_{r}}\left|\sum_{i=0}^{\infty}(-1)^{i} \frac{\Gamma(\alpha+1)}{i ! \Gamma(\alpha-i+1)} v_{k+i} x_{k+i}-L\right|^{p_{r}}=0\right\},
\end{aligned}
$$

where $v=\left(v_{k}\right)$ is a fixed sequence of nonzero real or complex numbers.

Theorem 10. Assume that $\left(p_{r}\right)$ is a bounded sequence. Then the sequence spaces $C\left(\Delta_{\nu}^{\alpha}, p\right)_{\theta}, C\left[\Delta_{\nu}^{\alpha}, p\right]_{\theta}, C_{\infty}\left(\Delta_{v}^{\alpha}, p\right)_{\theta}$, $C_{\infty}\left[\Delta_{\nu}^{\alpha}, p\right]_{\theta}$, and $N\left(\Delta_{v}^{\alpha}, p\right)_{\theta}$ are linear spaces.

Proof. Because the linearity may be proved in a similar way for each of the sets of sequences, hence it is omitted. 
Theorem 11. If $p=p_{r}$ for all $r \in \mathbb{N}$, then the sequence space $C\left[\Delta^{\alpha}{ }_{v}, p\right]_{\theta}$ is a BK-space with the norm defined by

$$
\begin{gathered}
\|x\|_{1}=\sum_{i=0}^{\infty}\left|v_{i} x_{i}\right|+\left\{\sum _ { r = 1 } ^ { \infty } \left(\frac{1}{h_{r}}\right.\right. \\
\left.\left.\quad \sum_{k \in I_{r}}\left|\sum_{i=0}^{\infty}(-1)^{i} \frac{\Gamma(\alpha+1)}{i ! \Gamma(\alpha-i+1)} v_{k+i} x_{k+i}\right|\right)^{p}\right\}^{1 / p},
\end{gathered}
$$

Also if $p_{r}=1$ for all $r \in \mathbb{N}$, then the sequence spaces $C_{\infty}\left[\Delta_{v}^{\alpha}\right.$, $p]_{\theta}$ and $N\left(\Delta_{v}^{\alpha}, p\right)_{2^{r}}$ are BK-spaces with the norm defined by

$$
\begin{aligned}
\|x\|_{2}= & \sum_{i=0}^{\infty}\left|v_{i} x_{i}\right| \\
& +\sup _{r} \frac{1}{h_{r}} \sum_{k \in I_{r}}\left|\sum_{i=0}^{\infty}(-1)^{i} \frac{\Gamma(\alpha+1)}{i ! \Gamma(\alpha-i+1)} v_{k+i} x_{k+i}\right| .
\end{aligned}
$$

Proof. We give the proof for the space $C_{\infty}\left[\Delta_{\nu}^{\alpha}, p\right]_{\theta}$ and that of others followed by using similar techniques.

Suppose $\left(x^{n}\right)$ is a Cauchy sequence in $C_{\infty}\left[\Delta_{v}^{\alpha}, p\right]_{\theta}$, where $x^{n}=\left(x_{j}^{n}\right)$ and $x^{m}=\left(x_{j}^{m}\right)$ are two elements in $C_{\infty}\left[\Delta_{\nu}^{\alpha}, p\right]_{\theta}$. Then there exists a positive integer $n_{0}(\epsilon)$ such that $\| x^{n}-$ $x^{m} \|_{2} \rightarrow 0$ as $m, n \rightarrow \infty$ for all $m, n \geq n_{0}(\epsilon)$ and for each $j \epsilon$ $\mathbb{N}$. Therefore $\left(x_{i}^{1}, x_{i}^{2}, \ldots\right)$ and $\left(\Delta_{\nu}^{\alpha}\left(x_{j}^{1}\right), \Delta_{\nu}^{\alpha}\left(x_{j}^{2}\right), \ldots\right)$ are Cauchy sequences in complex field $\mathbb{C}$ and $C_{\infty}\left[\Delta_{\nu}^{\alpha}, p\right]_{\theta}$, respectively. By using the completeness of $\mathbb{C}$ and $C_{\infty}\left[\Delta_{\nu}^{\alpha}, p\right]_{\theta}$, we have that they are convergent and suppose that $x_{i}^{n} \rightarrow x_{i}$ in $\mathbb{C}$ and $\left(\Delta_{v}^{\alpha}\left(x_{j}^{n}\right)\right) \rightarrow y_{j}$ in $C_{\infty}\left[\Delta_{v}^{\alpha}, p\right]_{\theta}$ for each $j \in \mathbb{N}$ as $n \rightarrow \infty$. Then we can find a sequence $\left(x_{j}\right)$ such that $y_{j}=\Delta_{v}^{\alpha}\left(x_{j}\right)$ for each $j \in \mathbb{N}$. These $x_{j}$ 's can be interpreted as

$$
\begin{aligned}
& x_{j}= \frac{1}{v_{j}} \sum_{i=1}^{j-k}(-1)^{r} \frac{\Gamma(j-i)}{(k-1) ! \Gamma(j-i-k+1)} y_{i} \\
&=\frac{1}{v_{j}} \sum_{i=1}^{j} \frac{(-1)^{r}}{(k-1) !} \frac{\Gamma(j+k-i)}{\Gamma(j-i+1)} y_{i-k}, \\
& \quad\left(y_{1-k}=y_{2-k}=\cdots=y_{0}=0\right)
\end{aligned}
$$

for sufficiently large $j$; that is, $j>2 k$. Then $\left(\Delta_{\gamma}^{\alpha}\left(x^{n}\right)\right)=$ $\left(\Delta_{v}^{\alpha}\left(x_{j}^{1}\right), \Delta_{v}^{\alpha}\left(x_{j}^{2}\right), \ldots\right)$ converges to $\left(\Delta_{v}^{\alpha}\left(x_{j}\right)\right)$ for each $j \in \mathbb{N}$ as $n \rightarrow \infty$. Thus $\left\|x^{n}-x\right\|_{2} \rightarrow 0$ as $m \rightarrow \infty$. Since $C_{\infty}\left[\Delta_{\nu}^{\alpha}, p\right]_{\theta}$ is a Banach space with continuous coordinates, that is, $\| x^{n}-$ $x \|_{2} \rightarrow 0$ implies $\left|x_{j}^{n}-x_{j}\right| \rightarrow 0$ for each $j \in \mathbb{N}$, as $n \rightarrow \infty$, this shows that $C_{\infty}\left[\Delta_{v}^{\alpha}, p\right]_{\theta}$ is a $B K$-space.
Theorem 12. If $p=p_{r}$ for all $r \in \mathbb{N}$, then the sequence space $C\left(\Delta_{v}^{\alpha}, p\right)_{\theta}$ is a BK-space with the norm defined by

$$
\begin{gathered}
\|x\|_{3}=\sum_{i=0}^{\infty}\left|v_{i} x_{i}\right|+\left\{\sum_{r=1}^{\infty} \mid \frac{1}{h_{r}}\right. \\
\left.\left.\cdot \sum_{k \in I_{r}} \sum_{i=0}^{\infty}(-1)^{i} \frac{\Gamma(\alpha+1)}{i ! \Gamma(\alpha-i+1)} v_{k+i} x_{k+i}\right|^{p}\right\}^{1 / p},
\end{gathered}
$$

$$
(1 \leq p)
$$

Also if $p_{r}=1$ for all $r \in \mathbb{N}$, then the sequence space $C_{\infty}\left(\Delta_{\nu}^{\alpha}, p\right)_{\theta}$ is a $B K$-space with the norm defined by

$$
\begin{aligned}
\|x\|_{4}= & \sum_{i=0}^{\infty}\left|v_{i} x_{i}\right| \\
& +\sup _{r}\left|\frac{1}{h_{r}} \sum_{k \in I_{r}} \sum_{i=0}^{\infty}(-1)^{i} \frac{\Gamma(\alpha+1)}{i ! \Gamma(\alpha-i+1)} v_{k+i} x_{k+i}\right| .
\end{aligned}
$$

Proof. The proof follows from Theorem 11.

Now, we can present the following theorem, determining some inclusion relations without proof, since it is a routine verification.

Theorem 13. Let two positive proper fractions $\alpha>\beta>0$ and $p_{r}=p$ for each $r \in \mathbb{N}$ be given. Then the following inclusions are satisfied:

(i) $C\left(\Delta_{v}^{\beta}, p\right)_{\theta} \subset C\left(\Delta_{v}^{\alpha}, p\right)_{\theta}$,

(ii) $C\left[\Delta_{v}^{\beta}, p\right]_{\theta} \subset C\left[\Delta_{v}^{\alpha}, p\right]_{\theta}$,

(iii) $C\left(\Delta_{v}^{\alpha}, p\right)_{\theta} \subset C\left(\Delta_{v}^{\alpha}, q\right)_{\theta},(0<p<q)$.

\section{Lacunary Statistical Convergence of Difference Sequences}

The concept of statistical convergence from different aspects has been studied by various mathematicians. The notion of statistical convergence was independently introduced by Fast [23] and Schoenberg [24].

Let $K$ be a subset of the set of natural numbers $\mathbb{N}$. Then the asymptotic density of $K$ denoted by $\delta(K)$ is defined as $\delta(K)=$ $\lim _{n}(1 / n)|\{k \leq n: k \in K\}|$, where the vertical bars denote the cardinality of the enclosed set.

A number sequence $x=\left(x_{k}\right)$ said to be statistically convergent to the number $L$ if, for each $\varepsilon>0$, the set $K(\varepsilon)=$ $\left\{k \leq n:\left|x_{k}-L\right|>\varepsilon\right\}$ has asymptotic density zero; that is, $\lim _{n}(1 / n)\left|\left\{k \leq n:\left|x_{k}-L\right| \geq \varepsilon\right\}\right|=0$.

Definition 14. Let $\theta=\left(k_{r}\right)$ be a lacunary sequence and $\alpha$ a proper fraction. Then the sequence $x=\left(x_{k}\right)$ is said to be $\Delta_{v^{-}}^{\alpha}$ lacunary statistically convergent to a real or complex number $L$ if, for each $\varepsilon>0$,

$$
\lim _{r \rightarrow \infty} \frac{1}{h_{r}}\left|\left\{k \in\left(k_{r-1}, k_{r}\right]:\left|\Delta_{\nu}^{\alpha}\left(x_{k}\right)-L\right| \geq \varepsilon\right\}\right|=0,
$$


where the vertical bars denote the cardinality of the enclosed set. The set of $\Delta_{v}^{\alpha}$-lacunary statistically convergent sequences will be denoted by $\Delta_{\gamma}^{\alpha}\left(S_{\theta}\right)$. In this case we write $x_{k} \rightarrow$ $L\left(\Delta_{\nu}^{\alpha}\left(S_{\theta}\right)\right)$.

In particular, the $\Delta_{v}^{\alpha}$-lacunary statistical convergence includes many special cases; that is, in the case $\alpha=m \in$ $\mathbb{N}, \Delta_{v}^{\alpha}$-lacunary statistical convergence reduces to the $\Delta_{v}^{m}$ lacunary statistical convergence defined by [15].

Theorem 15. Let $0<p<\infty$. If $x_{k} \rightarrow L\left(N\left(\Delta_{v}^{\alpha}, p\right)_{\theta}\right)$ for $p_{r}=p$, then $x_{k} \rightarrow L\left(\Delta_{v}^{\alpha}\left(S_{\theta}\right)\right)$. If $x \in \Delta_{v}^{\alpha}\left(\ell_{\infty}\right)$ and $x_{k} \rightarrow$ $L\left(\Delta_{\nu}^{\alpha}\left(S_{\theta}\right)\right)$, then $x_{k} \rightarrow L\left(N\left(\Delta_{v}^{\alpha}, p\right)_{\theta}\right)$.

Proof. Let $x=\left(x_{k}\right) \in L\left(N\left(\Delta_{v}^{\alpha}, p\right)_{\theta}\right)$ and $\epsilon>0$ and let $\sum_{*}$ denote the sum over $k \in\left(k_{r-1}, k_{r}\right]$ such that $\left|\Delta_{\nu}^{\alpha}\left(x_{k}\right)-L\right| \geq \varepsilon$. We have that

$$
\begin{aligned}
& \frac{1}{h_{r}} \sum_{*}\left|\Delta_{v}^{\alpha} x_{k}-L\right|^{p} \\
& \quad \geq \frac{1}{h_{r}}\left|\left\{k \in\left(k_{r-1}, k_{r}\right]:\left|\Delta^{\alpha}\left(x_{k}\right)-L\right| \geq \varepsilon\right\}\right| \epsilon^{p} .
\end{aligned}
$$

So we observe, by passing to limit as $r \rightarrow \infty$, in (18)

$$
\begin{gathered}
\lim _{r \rightarrow \infty} \frac{1}{h_{r}}\left|\left\{k \in\left(k_{r-1}, k_{r}\right]:\left|\Delta_{v}^{\alpha}\left(x_{k}\right)-L\right| \geq \varepsilon\right\}\right| \\
\leq \frac{1}{\epsilon^{p}}\left(\lim _{r \rightarrow \infty} \frac{1}{h_{r}} \sum_{*}\left|\Delta_{v}^{\alpha} x_{k}-L\right|^{p}\right)=0
\end{gathered}
$$

which implies that $x_{k} \rightarrow L\left(\Delta_{\gamma}^{\alpha}\left(S_{\theta}\right)\right)$.

Suppose that $x \in \Delta_{\nu}^{\alpha}\left(\ell_{\infty}\right)$ and $x_{k} \rightarrow L\left(\Delta_{\nu}^{\alpha}(S)\right)$. Then it is obvious that $\left(\Delta_{v}^{\alpha} x\right) \in \ell_{\infty}$ and $\left(1 / h_{r}\right) \mid\left\{k \in\left(k_{r-1}, k_{r}\right]: \mid \Delta_{\nu}^{\alpha}\left(x_{k}\right)-\right.$ $L \mid \geq \varepsilon\} \mid \rightarrow 0$ as $r \rightarrow \infty$. Let $\varepsilon>0$ be given and there exists $n_{0} \in \mathbb{N}$ such that

$$
\begin{aligned}
& \frac{\left|\left\{k \in\left(k_{r-1}, k_{r}\right]:\left|\Delta^{\alpha}\left(x_{k}\right)-L\right| \geq(\varepsilon / 2)^{1 / p}\right\}\right|}{h_{r}} \\
& \quad \leq \frac{\varepsilon}{2\left(\left\|\Delta_{\nu}^{\alpha} x\right\|_{\infty}+L\right)^{p}},
\end{aligned}
$$

where $\sum_{i=1}^{\infty}\left|v_{i} x_{i}\right|=L$, for all $r>n_{0}$. Furthermore, we can write

$$
\begin{aligned}
\left|\Delta_{\nu}^{\alpha}\left(x_{k}\right)-L\right| & \leq\left\|\Delta_{\nu}^{\alpha}\left(x_{k}\right)-L\right\|_{\Delta_{\nu}^{\alpha}} \leq L+\left\|\Delta_{\nu}^{\alpha} x\right\|_{\infty} \\
& =\|x\|_{\Delta_{\nu}^{\alpha}(X)} .
\end{aligned}
$$

For $r>n_{0}$,

$$
\begin{aligned}
& \frac{1}{h_{r}} \sum_{k \in I_{r}}\left|\Delta_{v}^{\alpha} x_{k}-L\right|^{p} \\
& \quad=\frac{1}{h_{r}}\left(\sum_{k \in L_{r}}\left|\Delta^{\alpha} x_{k}-L\right|^{p}+\sum_{k \notin L_{r}}\left|\Delta^{\alpha} x_{k}-L\right|^{p}\right) \\
& \quad<\frac{1}{h_{r}}\left(h_{r} \frac{\varepsilon}{2}+h_{r} \frac{\varepsilon\|x\|_{\Delta_{v}^{\alpha}(X)}^{p}}{2\|x\|_{\Delta_{v}^{\alpha}(X)}^{p}}\right)=\varepsilon,
\end{aligned}
$$

where $L_{r}=\left\{k \in\left(k_{r-1}, k_{r}\right]:\left|\Delta^{\alpha}\left(x_{k}\right)-L\right| \geq(\varepsilon / 2)^{1 / p}\right\}$. Hence $x_{k} \rightarrow L\left(N\left(\Delta_{v}^{\alpha}, p\right)_{\theta}\right)$. This completes the proof.

Corollary 16. The following statements hold:

(a) $S \cap \ell_{\infty} \subset \Delta_{\nu}^{\alpha}\left(S_{\theta}\right) \cap \Delta_{\nu}^{\alpha}\left(\ell_{\infty}\right)$,

(b) $\Delta_{v}^{\alpha}\left(S_{\theta}\right) \cap \Delta_{v}^{\alpha}\left(\ell_{\infty}\right)=\Delta_{v}^{\alpha}\left(\omega_{p}\right)$.

Definition 17. Let $\theta$ be a lacunary sequence and $\alpha$ a proper fraction. Then a sequence $x=\left(x_{k}\right)$ is said to be $\Delta_{v}^{\alpha}$-lacunary statistically Cauchy if there exists a number $N=N(\varepsilon)$ such that

$$
\lim _{r \rightarrow \infty} \frac{1}{h_{r}}\left|\left\{k \in\left(k_{r-1}, k_{r}\right]:\left|\Delta_{v}^{\alpha}\left(x_{k}-x_{N}\right)\right| \geq \varepsilon\right\}\right|=0
$$

for every $\varepsilon>0$.

Theorem 18. If $x=\left(x_{k}\right)$ is a $\Delta_{v}^{\alpha}$-lacunary statistically convergent sequence, then $x$ is a $\Delta_{v}^{\alpha}$-lacunary statistically Cauchy sequence.

Proof. Assume that $x_{k} \rightarrow L\left(\Delta_{\gamma}^{\alpha}\left(S_{\theta}\right)\right)$ and $\varepsilon>0$. Then $\left|\Delta_{\nu}^{\alpha}\left(x_{k}\right)-L\right|<\varepsilon / 2$ for almost all $k$, and if we select $N$, then $\left|\Delta_{\gamma}^{\alpha}\left(x_{N}\right)-L\right|<\varepsilon / 2$ holds. Now, we have

$$
\begin{aligned}
\left|\Delta_{v}^{\alpha}\left(x_{k}\right)-\Delta_{v}^{\alpha}\left(x_{N}\right)\right| & <\left|\Delta_{v}^{\alpha}\left(x_{k}\right)-L\right|+\left|\Delta_{v}^{\alpha}\left(x_{N}\right)-L\right| \\
& <\varepsilon
\end{aligned}
$$

for almost all $k$. Hence $x$ is a $\Delta_{v}^{\alpha}$-lacunary statistically Cauchy sequence.

Theorem 19. Let $\alpha$ be a proper fraction and $0<\inf p_{r} \leq p_{r} \leq$ $\sup p_{r}<\infty$. Then $N\left(\Delta_{v}^{\alpha}, p\right)_{\theta} \subset \Delta_{\nu}^{\alpha}\left(S_{\theta}\right)$.

Proof. Suppose that $x=\left(x_{k}\right) \in N\left(\Delta_{v}^{\alpha}, p\right)_{\theta}$ and $\sum_{*}$ denote the sum over $k \in\left(k_{r-1}, k_{r}\right]$ such that $\left|\Delta_{\nu}^{\alpha}\left(x_{k}\right)-L\right| \geq \varepsilon$. Therefore we have

$$
\begin{aligned}
& \frac{1}{h_{r}} \sum_{k \in I_{r}}\left|\Delta_{v}^{\alpha}\left(x_{k}\right)-L\right|^{p_{r}} \geq \frac{1}{h_{r}} \sum_{*}\left|\Delta^{\alpha} x_{k}-L\right|^{p_{r}} \\
& \quad \geq \frac{1}{h_{r}} \sum_{*} \varepsilon^{\inf p_{r}} \\
& \quad \geq \frac{1}{h_{r}}\left|\left\{k \in I_{r}:\left|\Delta^{\alpha}\left(x_{k}\right)-L\right| \geq \varepsilon\right\}\right| \varepsilon^{\inf p_{r}} .
\end{aligned}
$$

Taking the limit as $r \rightarrow \infty$,

$$
\begin{aligned}
& \lim _{r \rightarrow \infty} \frac{1}{h_{r}}\left|\left\{k \in I_{r}:\left|\Delta_{\nu}^{\alpha}\left(x_{k}\right)-L\right| \geq \varepsilon\right\}\right| \\
& \leq \frac{1}{\varepsilon^{\inf p_{r}}}\left(\lim _{r \rightarrow \infty} \frac{1}{h_{r}} \sum_{k \in I_{r}}\left|\Delta_{\nu}^{\alpha} x_{k}-L\right|^{p_{r}}\right)=0
\end{aligned}
$$

implies that $x \in \Delta_{\nu}^{\alpha}\left(S_{\theta}\right)$. Hence $N\left(\Delta_{\nu}^{\alpha}, p\right)_{\theta} \subset \Delta_{v}^{\alpha}\left(S_{\theta}\right)$. 


\section{Concluding Remarks}

In this paper, certain results on some lacunary statistical difference sequence spaces of order $m(m \in \mathbb{N})$ have been extended to the difference sequence spaces of fractional order $\alpha$. The results presented in this paper not only generalize the earlier works done by several authors $[2,3,15,20,21]$ but also give a new perspective regarding the development of difference sequences. As a future work we will study certain matrix transformations of these spaces.

\section{Conflict of Interests}

The author declares that there is no conflict of interests regarding the publication of this paper.

\section{Acknowledgment}

The author expresses his sincere thanks to the referees for their valuable suggestions and comments, which improved the presentation of this paper.

\section{References}

[1] H. Kizmaz, "On certain sequence spaces," Canadian Mathematical Bulletin, vol. 24, no. 2, pp. 169-176, 1981.

[2] M. Et and R. Colak, "On some generalized difference sequence spaces," Soochow Journal of Mathematics, vol. 21, no. 4, pp. 377386, 1995.

[3] M. Et and A. Esi, "On Köthe-Toeplitz duals of generalized difference sequence spaces," Bulletin of the Malaysian Mathematical Sciences Society, vol. 23, no. 1, pp. 25-32, 2000.

[4] M. Et and M. Basarir, "On some new generalized difference sequence spaces," Periodica Mathematica Hungarica, vol. 35, no. 3, pp. 169-175, 1997.

[5] C. Aydın and F. Başar, "Some new difference sequence spaces," Applied Mathematics and Computation, vol. 157, no. 3, pp. 677693, 2004.

[6] M. Mursaleen, "Generalized spaces of difference sequences," Journal of Mathematical Analysis and Applications, vol. 203, no. 3, pp. 738-745, 1996.

[7] M. Mursaleen and A. K. Noman, "On some new difference sequence spaces of non-absolute type," Mathematical and Computer Modelling, vol. 52, no. 3-4, pp. 603-617, 2010.

[8] E. Malkowsky, M. Mursaleen, and S. Suantai, “The dual spaces of sets of difference sequences of order $m$ and matrix transformations," Acta Mathematica Sinica (English Series), vol. 23, no. 3, pp. 521-532, 2007.

[9] Ç. A. Bektaş, M. Et, and R. Çolak, "Generalized difference sequence spaces and their dual spaces," Journal of Mathematical Analysis and Applications, vol. 292, no. 2, pp. 423-432, 2004.

[10] B. C. Tripathy, Y. Altin, and M. Et, "Generalized difference sequence spaces on seminormed space defined by Orlicz functions," Mathematica Slovaca, vol. 58, no. 3, pp. 315-324, 2008.

[11] B. C. Tripathy and B. Sarma, "Some classes of difference paranormed sequence spaces defined by Orlicz functions," Thai Journal of Mathematics, vol. 3, no. 2, pp. 209-218, 2005.

[12] G. Das and S. K. Mishra, "Banach limits and lacunary strong almost convergence," Journal of Orissa Mathematical Society, vol. 2, no. 2, pp. 61-70, 1983.
[13] R. Colak, B. C. Tripathy, and M. Et, "Lacunary strongly summable sequences and $q$-lacunary almost statistical convergence," Vietnam Journal of Mathematics, vol. 34, no. 2, pp. 129$138,2006$.

[14] V. Karakaya, "Some geometric properties of sequence spaces involving lacunary sequence," Journal of Inequalities and Applications, vol. 2007, Article ID 081028, 8 pages, 2007.

[15] M. Et, "Generalized Cesáro difference sequence spaces of nonabsolute type involving lacunary sequences," Applied Mathematics and Computation, vol. 219, no. 17, pp. 9372-9376, 2013.

[16] J. S. Shiue, “On the Cesáro sequence space," Tamkang Journal of Mathematics, vol. 1, no. 1, pp. 19-25, 1970.

[17] A. A. Jagers, "A note on Cesáro sequence spaces," Nieuw Archief voor Wiskunde, vol. 22, no. 3, pp. 113-124, 1974.

[18] P. N. Ng and P. Y. Lee, "Cesáro sequence spaces of non-absolute type," Commentationes Mathematicae, vol. 20, pp. 429-433, 1978.

[19] P. N. Ng and P. Y. Lee, "On the associate spaces of Cesáro sequence space," Nanta Mathematica, vol. 9, no. 2, pp. 168-170, 1976.

[20] P. Baliarsingh, "Some new difference sequence spaces of fractional order and their dual spaces," Applied Mathematics and Computation, vol. 219, no. 18, pp. 9737-9742, 2013.

[21] P. Baliarsingh and S. Dutta, "On the classes of fractional order difference sequence spaces and their matrix transformations," Applied Mathematics and Computation, vol. 250, pp. 665-674, 2015.

[22] U. Kadak and P. Baliarsingh, "On certain Euler difference sequence spaces of fractional order and related dual properties," The Journal of Nonlinear Science and Applications, vol. 8, pp. 997-1004, 2015.

[23] H. Fast, "Sur la convergence statistique," Colloquium Mathematicum, vol. 2, no. 1, pp. 241-244, 1951.

[24] I. J. Schoenberg, "The integrability of certain functions and related summability methods," The American Mathematical Monthly, vol. 66, pp. 361-375, 1959. 


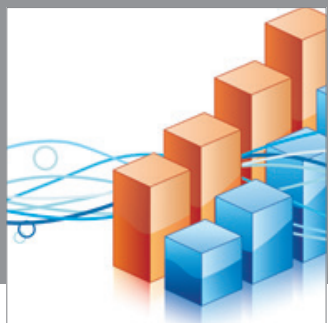

Advances in

Operations Research

mansans

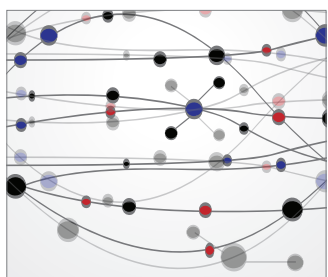

The Scientific World Journal
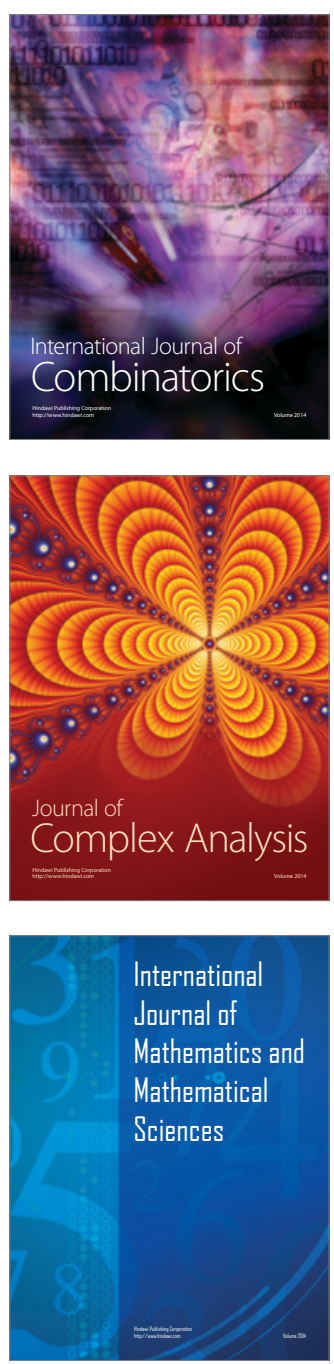
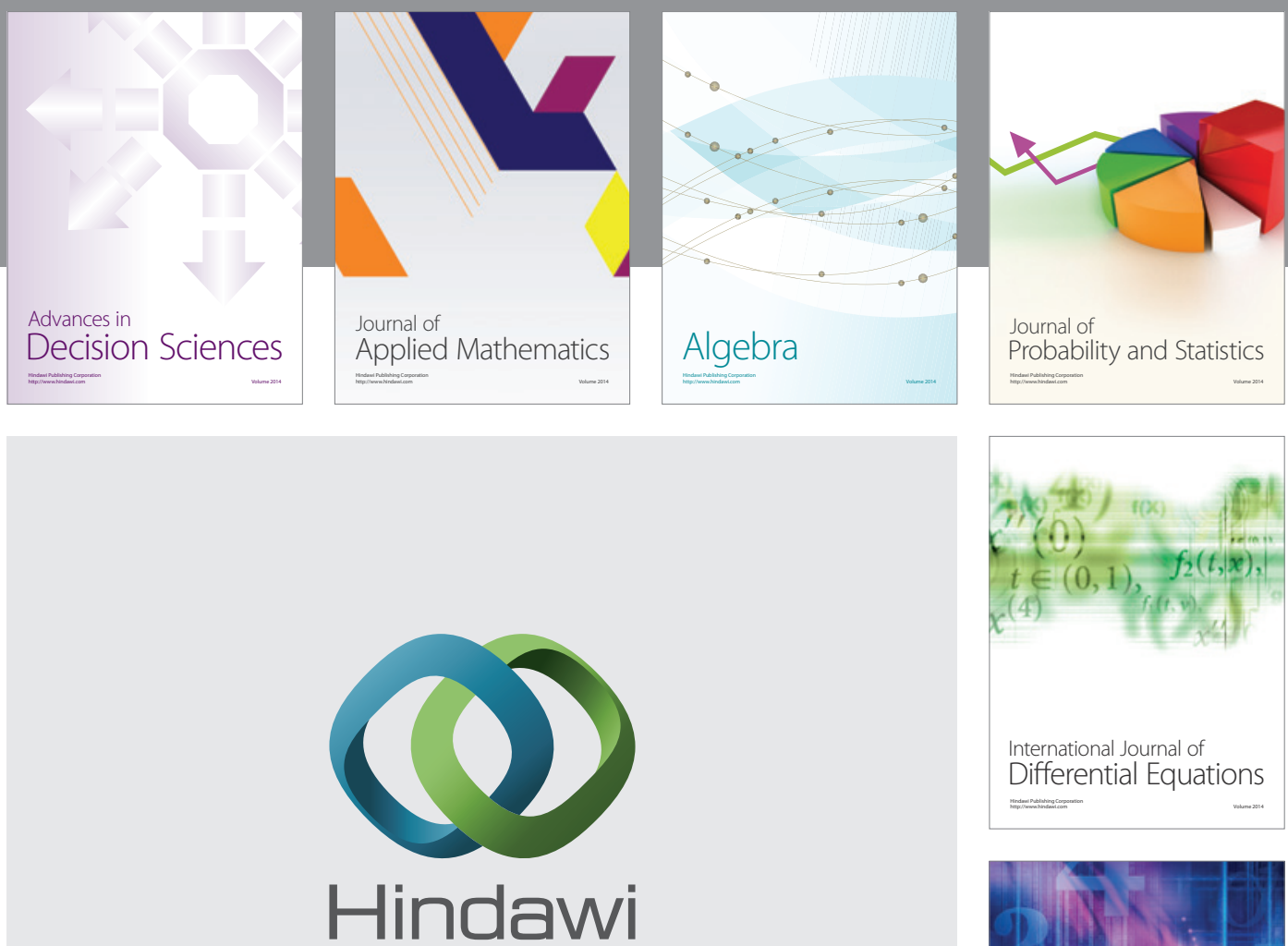

Submit your manuscripts at http://www.hindawi.com
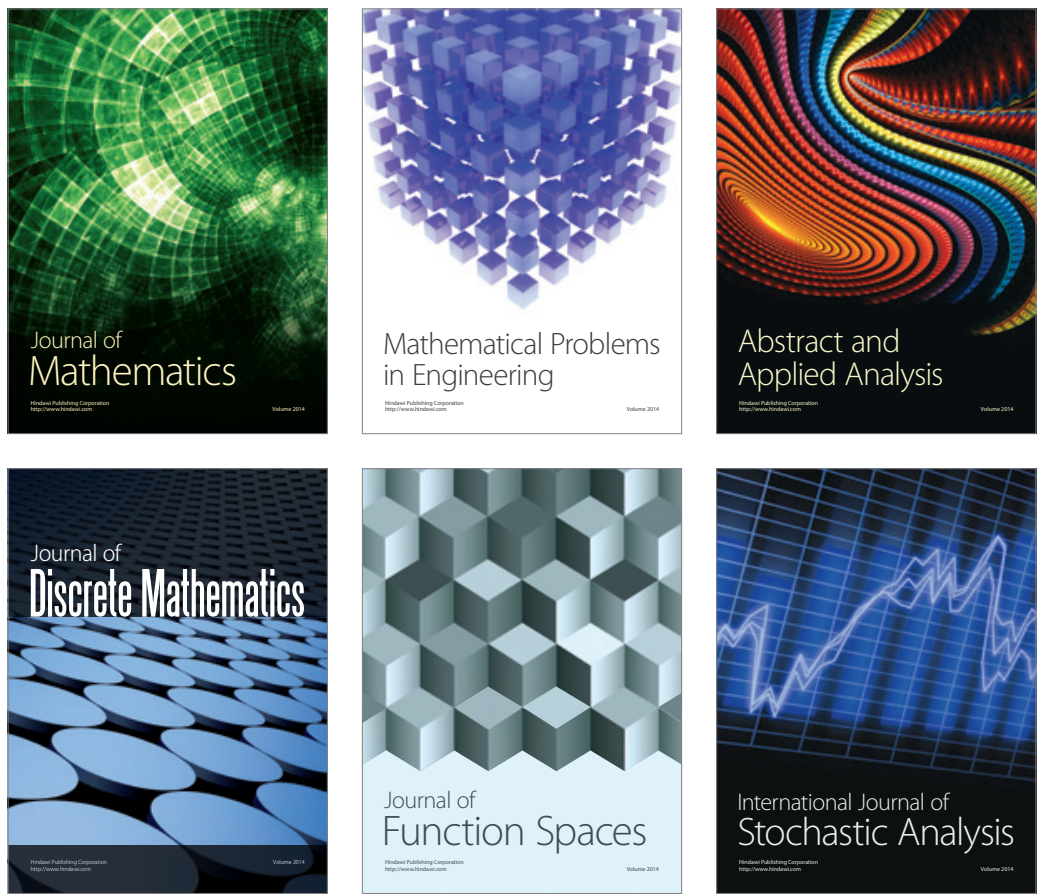

Journal of

Function Spaces

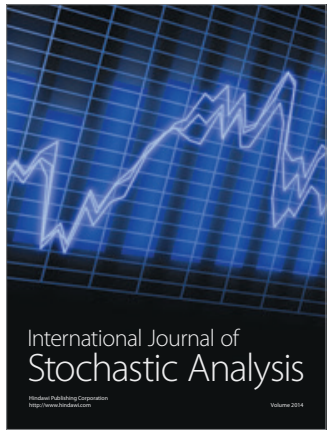

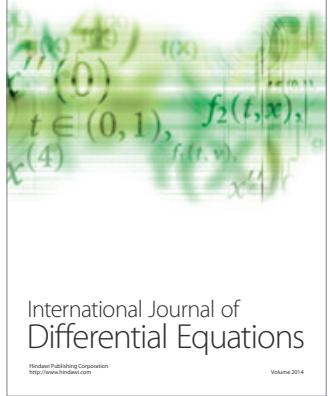
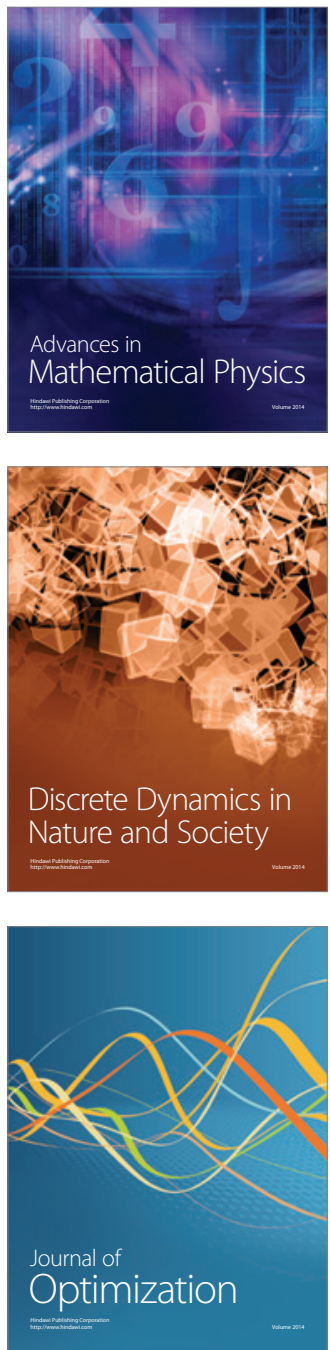